TITLE:

\title{
Precursor of color superconductivity in hot quark matter
}

\section{$\operatorname{AUTHOR}(\mathrm{S}):$}

Kitazawa, M; Koide, T; Kunihiro, T; Nemoto, Y

\section{CITATION:}

Kitazawa, M ...[et al]. Precursor of color superconductivity in hot quark matter. PHYSICAL REVIEW D 2002, 65(9): 091504.

ISSUE DATE:

2002-05-01

URL:

http://hdl.handle.net/2433/50051

RIGHT:

Copyright 2002 American Physical Society 
PHYSICAL REVIEW D, VOLUME 65, 091504(R)

\title{
Precursor of color superconductivity in hot quark matter
}

\author{
M. Kitazawa, ${ }^{1}$ T. Koide, ${ }^{2}$ T. Kunihiro, ${ }^{2}$ and Y. Nemoto ${ }^{2, *}$ \\ ${ }^{1}$ Department of Physics, Kyoto University, Kyoto 606-8502, Japan \\ ${ }^{2}$ Yukawa Institute for Theoretical Physics, Kyoto University, Kyoto 606-8502, Japan
}

(Received 7 November 2001; published 17 May 2002)

\begin{abstract}
We investigate the possible precursory phenomena of color superconductivity in quark matter at finite temperature $T$ with use of a low-energy effective theory of QCD. It is found that the fluctuating pair field exists with a prominent strength even well above the critical temperature $T_{c}$. We show that the collective pair field has a complex energy located in the second Riemann sheet, which approaches the origin as $T$ is lowered to $T_{c}$. We discuss the possible relevance of the precursor to the observables to be detected in heavy ion collisions.
\end{abstract}

DOI: 10.1103/PhysRevD.65.091504

PACS number(s): 12.38.Mh, 11.15.Ex, 25.75.-q, 74.40.+k

It is an intriguing subject to explore how the QCD vacuum changes in hot and dense hadronic matter [1,2]. The recent lattice QCD simulations [3] show that the QCD vacuum undergoes a phase transition to a chirally restored and deconfined phase at low temperature $(T)$ as $T$ $\sim 170 \mathrm{MeV}$. Although it is still a great challenge to perform a Monte Carlo simulation with a finite chemical potential $\mu$ on lattice QCD with an SU(3) color group, we believe that a chirally restored and deconfined system is realized in dense hadronic matter with large $\mu$.

It is to be noticed that a system with a finite $\mu$ at low $T$ can have a Fermi surface and the system may be described as a Fermi liquid composed of quarks. In accordance with the validity of the diquark-quark picture of baryons [4], the quark-quark interaction is attractive in some specific channels. Then the existence of the Fermi surface gives rise to a Cooper instability with respect to the formation of the diquark or Cooper pair in the most attractive channel, and the system is rearranged to a superconducting phase where the color-gauge symmetry is dynamically broken [5]. Such color superconductivity (CSC) has recently acquired renewed interest, because the resulting gap $\Delta$ was shown to be as large as $100 \mathrm{MeV}$ [6,7]. Furthermore, the color and flavor degrees of freedom of quarks give a fantastically rich structure in dense quark matter with CSC [8]: Such quark matter may be realized in the deep interior of neutron stars (NS), and characteristic phenomena observed for NS such as the glitch phenomena might be attributed to the existence of the CSC in the NS.

Can CSC be relevant to experiments in the laboratory on Earth? The experiment using the available facilities, such as the BNL Relativistic Heavy Ion Collider (RHIC) using high energy heavy ion collisions seems unfortunately unfit for the problem, because the matter produced by RHIC is almost baryon-free with much higher temperatures than the critical temperature $T_{c} \sim 50-60 \mathrm{MeV}$ of CSC. The purpose of the present paper is to explore possible precursory phenomena of CSC at $T>T_{c}$, i.e., in the Wigner phase and show that the fluctuation of the pair field is significant at $T$ well above $T_{c}$,

\footnotetext{
*Present address: RIKEN BNL Research Center, Brookhaven National Laboratory, Upton, NY 11973.
}

which thereby might affect observables to be detected by the heavy ion collisions with large baryon stopping [9].

Since we are interested in relatively the low $T$ and $\rho$ region, we adopt a low-energy effective theory of QCD as was done in $[6,10]$ where an instanton-induced interaction is employed. We remark here that a simplified version of the instanton-induced interaction in the two-flavor case takes the form of the Nambu-Jona-Lasinio (NJL) model [12], which can be also derived by a Fierz transformation of the onegluon exchange interaction with a heavy-gluon approximation; see $[1,4,13,14]$. Indeed it was shown later [11] that the physical content given in [10] can be nicely reproduced by the simple NJL model. Therefore, we simply take the following NJL model as an effective theory:

$$
\begin{aligned}
\mathcal{L}= & \bar{\psi} i \not \partial \psi+G_{S}\left[(\bar{\psi} \psi)^{2}+\left(\bar{\psi} i \gamma_{5} \vec{\tau} \psi\right)^{2}\right]+G_{C}\left(\bar{\psi} i \gamma_{5} \tau_{2} \lambda_{2} \psi^{C}\right) \\
& \times\left(\bar{\psi}^{C} i \gamma_{5} \tau_{2} \lambda_{2} \psi\right)
\end{aligned}
$$

where $\psi^{C} \equiv C \bar{\psi}^{T}$, with $C=i \gamma^{2} \gamma^{0}$ being the charge conjugation operator. Here we have confined ourselves in the twoflavor case in the chiral limit; $\tau_{2}$ and $\lambda_{2}$ are the second components of the Pauli and Gell-Mann matrices representing the flavor $\mathrm{SU}(2)$ and color $\mathrm{SU}(3)$, respectively. The scalar coupling constant $G_{S}$ and the three-dimensional momentum cutoff $\Lambda$ are determined so that the pion decay constant $f_{\pi}=93 \mathrm{MeV}$ and the chiral condensate $\langle\bar{\psi} \psi\rangle$ $=(-250 \mathrm{MeV})^{3}$ are reproduced in the chiral limit [13]; $G_{S}=5.01 \mathrm{GeV}^{-2}$ and $\Lambda=650 \mathrm{MeV}$. As for the diquark coupling constant $G_{C}$, we shall fix $G_{C}$ at $3.11 \mathrm{GeV}^{-2}$ [11] in this paper. We have checked that the qualitative features of the results do not change for $G_{C}$ varied in the range used in the literature $[8,11]$.

The phase diagram determined in the mean-field approximation with Eq. (1) is shown in Fig. 1: The dashed line denotes the critical line for a second order phase transition and the solid line denotes the first order phase transition. The critical temperature of the chiral transition at $\mu=0$ is $T$ $=185 \mathrm{MeV}$, while the critical chemical potential of chiralCSC transition at $T=0$ is $\mu=316 \mathrm{MeV}$. The tricritical point where the order of the chiral transition changes from second to first order is located at about $(T, \mu)=(70,280) \mathrm{MeV}$. This phase diagram essentially coincides with those given in $[10,11]$. 


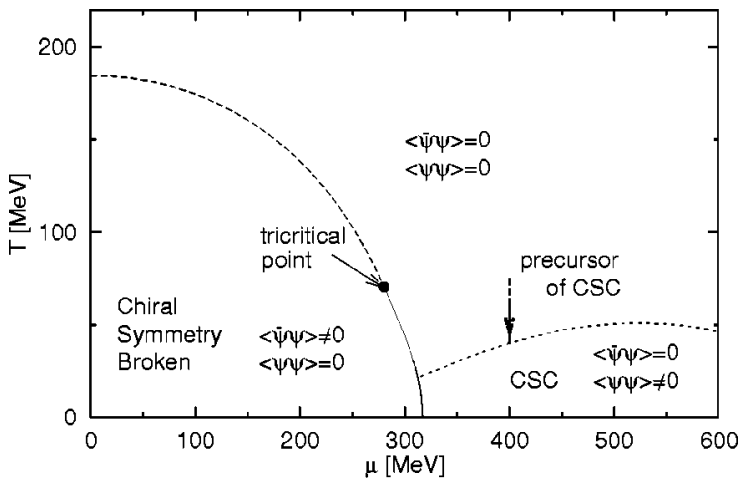

FIG. 1. The calculated phase diagram in a $T$ - $\mu$ plane in our model. The solid and dashed lines denote the critical line of a first and second order phase transition, respectively.

Given the phase diagram for the equilibrium state, we now proceed to examine fluctuations of the color Cooper pairs in the normal phase at $T>T_{c}$. We shall obtain possible collective states and their strength functions in the linear response theory. We suppose that the following external pair field is applied to the system: $\Delta_{e x}^{*}(x)=\Delta_{e x}^{*} e^{i \mathbf{k} \cdot \mathbf{x}} e^{-i \omega_{n} \tau}$, which is tantamount to adding the external Hamiltonian $H_{e x}=\int d \mathbf{x} \Delta_{e x}^{*}(x)\left(\bar{\psi}^{C} i \gamma_{5} \tau_{2} \lambda_{2} \psi\right)+$ h.c.. Then a color Cooperpair field is dynamically induced, as given by $\Delta_{i n d}^{*}(\mathbf{x}, \tau)$ $=-2 G_{C}\left\langle\bar{\psi}(\mathbf{x}, \tau) \tau_{2} \lambda_{2} i \gamma_{5} \psi^{C}(\mathbf{x}, \tau)\right\rangle$, where the angular brackets denote a statistical average. Using the anomalous Green function defined by $F^{\dagger}\left(\mathbf{x}, \tau ; \mathbf{x}^{\prime}, \tau^{\prime}\right)$ $=-\left\langle T_{\tau}\left\{\psi^{C}(\mathbf{x}, \tau) \bar{\psi}\left(\mathbf{x}^{\prime}, \tau^{\prime}\right)\right\}\right\rangle$, the induced pair field is ex- pressed as

$$
\Delta_{\text {ind }}^{*}(\mathbf{x}, \tau)=-2 G_{C} \operatorname{Tr} \tau_{2} \lambda_{2} i \gamma_{5} F^{\dagger}\left(\mathbf{x}, \tau ; \mathbf{x}, \tau_{+}\right)
$$

where $\operatorname{Tr}$ denotes the trace taken over the color, flavor, and spinor indices. $F^{\dagger}\left(\mathbf{x}, \tau ; \mathbf{x}^{\prime}, \tau^{\prime}\right)$ satisfies the following differential equation:

$$
\begin{array}{r}
{\left[-\gamma^{0} \frac{\partial}{\partial \tau}-K_{0}-\mu \gamma^{0}\right] F^{\dagger}\left(\mathbf{x}, \tau ; \mathbf{x}^{\prime}, \tau^{\prime}\right)} \\
=\Delta_{\text {tot }}^{*}(\mathbf{x}, \tau) \tau_{2} \lambda_{2} i \gamma_{5} G\left(\mathbf{x}, \tau ; \mathbf{x}^{\prime}, \tau^{\prime}\right),
\end{array}
$$

where $K_{0}=-i \vec{\gamma} \cdot \vec{\nabla}+M \quad$ and $\quad M=-2 G_{S}\langle\bar{\psi}(\mathbf{x}, \tau) \psi(\mathbf{x}, \tau)\rangle$ is the dynamical mass. Here, $\Delta_{t o t}^{*}(\mathbf{x}, \tau)=\Delta_{e x}^{*}(\mathbf{x}, \tau)$ $+\Delta_{\text {ind }}^{*}(\mathbf{x}, \tau)$ is the total pair field to induce the pair field: Notice the self-consistent nature of the problem. $G\left(\mathbf{x}, \tau ; \mathbf{x}^{\prime}, \tau^{\prime}\right)$ is the usual nonanomalous Matsubara Green function. To see a linear response of the system, one may replace $G\left(\mathbf{x}, \tau ; \mathbf{x}^{\prime}, \tau^{\prime}\right)$ in Eq. (3) with a free Matsubara Green function $G^{(0)}\left(\mathbf{x}, \tau ; \mathbf{x}^{\prime}, \tau^{\prime}\right)$; thus we have $F^{\dagger}\left(\mathbf{x}, \tau ; \mathbf{x}^{\prime}, \tau_{+}\right)=\left(-\gamma^{0}(\partial / \partial \tau)-K_{0}-\mu \gamma^{0}\right)^{-1}$ $\times \Delta_{\text {tot }}^{*}(\mathbf{x}, \tau) \tau_{2} \lambda_{2} i \gamma_{5} G^{(0)}\left(\mathbf{x}, \tau ; \mathbf{x}, \tau_{+}\right)$. Inserting this expression into Eq. (2) and performing the Fourier transformation, we have

$$
\Delta_{\text {ind }}^{*}\left(\mathbf{k}, \omega_{n}\right) \equiv-G_{C} \Delta_{\text {tot }}^{*}\left(\mathbf{k}, \omega_{n}\right) \mathcal{Q}\left(\mathbf{k}, \omega_{n}\right),
$$

where

$$
\begin{aligned}
\mathcal{Q}\left(\mathbf{k}, \omega_{n}\right)= & -2 N_{f}\left(N_{c}-1\right) \int \frac{d \mathbf{k}^{\prime}}{(2 \pi)^{3}} \frac{1}{E_{\mathbf{k}^{\prime}} E_{\mathbf{k}-\mathbf{k}^{\prime}}}\left[2 \left\{f^{-}\left(E_{\mathbf{k}^{\prime}}\right)\left[1-f^{+}\left(E_{\mathbf{k}-\mathbf{k}^{\prime}}\right)\right]-f^{+}\left(E_{\mathbf{k}-\mathbf{k}^{\prime}}\right)\right.\right. \\
& \left.\times\left[1-f^{-}\left(E_{\mathbf{k}^{\prime}}\right)\right]\right\} \frac{-E_{\mathbf{k}^{\prime}} E_{\mathbf{k}-\mathbf{k}^{\prime}}-\mathbf{k}^{\prime} \cdot\left(\mathbf{k}-\mathbf{k}^{\prime}\right)+M^{2}}{-i \omega_{n}-2 \mu-E_{\mathbf{k}-\mathbf{k}^{\prime}}+E_{\mathbf{k}^{\prime}}}-\left[1-f^{-}\left(E_{\mathbf{k}^{\prime}}\right)-f^{-}\left(E_{\mathbf{k}-\mathbf{k}^{\prime}}\right)\right] \frac{-E_{\mathbf{k}^{\prime}} E_{\mathbf{k}-\mathbf{k}^{\prime}}+\mathbf{k}^{\prime} \cdot\left(\mathbf{k}-\mathbf{k}^{\prime}\right)-M^{2}}{-i \omega_{n}-2 \mu+E_{\mathbf{k}-\mathbf{k}^{\prime}}+E_{\mathbf{k}^{\prime}}} \\
& \left.+\left[1-f^{+}\left(E_{\mathbf{k}^{\prime}}\right)-f^{+}\left(E_{\mathbf{k}-\mathbf{k}^{\prime}}\right)\right] \frac{-E_{\mathbf{k}^{\prime}} E_{\mathbf{k}-\mathbf{k}^{\prime}}+\mathbf{k}^{\prime} \cdot\left(\mathbf{k}-\mathbf{k}^{\prime}\right)-M^{2}}{-i \omega_{n}-2 \mu-E_{\mathbf{k}-\mathbf{k}^{\prime}}-E_{\mathbf{k}^{\prime}}}\right] .
\end{aligned}
$$

Here, $E_{\mathbf{k}}=\sqrt{\mathbf{k}^{2}+M^{2}}$ and $f^{ \pm}(E)=\{\exp [\beta(E \pm \mu)]+1\}^{-1}$ with $\beta=T^{-1}$. The first term in the brace on the right-hand side (rhs) of Eq. (5) is originated from the process $\Delta_{\text {tot }}^{*}+q \rightarrow \bar{q}$ and its inverse process $\bar{q} \rightarrow \Delta_{\text {tot }}^{*}+q$. The second and third terms can be interpreted similarly in terms of kinetic processes. Inserting $\Delta_{\text {tot }}^{*}\left(\mathbf{k}, \omega_{n}\right)=\Delta_{\text {ex }}^{*}\left(\mathbf{k}, \omega_{n}\right)+\Delta_{\text {ind }}^{*}\left(\mathbf{k}, \omega_{n}\right)$ into Eq. (4), we obtain the induced pair field in the linear response to the external field in the imaginary time as

$$
\Delta_{i n d}^{*}\left(\mathbf{k}, \omega_{n}\right)=\frac{-G_{C} \mathcal{Q}\left(\mathbf{k}, \omega_{n}\right)}{1+G_{C} \mathcal{Q}\left(\mathbf{k}, \omega_{n}\right)} \Delta_{e x}^{*} \equiv \mathcal{D}\left(\mathbf{k}, i \omega_{n}\right) \Delta_{e x}^{*} .
$$

This is a central result in the present work, on which the rest of the discussion is based. The proportionality constant in Eq. (6) converted in the real-time domain is called the response function, or retarded Green's function $D^{R}(\mathbf{k}, \omega)$, which is obtained by the analytic continuation of $\mathcal{D}(\mathbf{k}, \omega)$ to the upper half of the complex energy plane as $D^{R}(\mathbf{k}, \omega)$ $=\mathcal{D}(\mathbf{k}, \omega+i \eta)$.

Setting $M=0$, let us examine precursory collective modes above the CSC phase when approaching the critical point as shown by the arrow in Fig. 1. Then we notice the following equality holds:

$$
1+\left.G_{C} \mathcal{Q}(\mathbf{0}, 0)\right|_{T=T_{c}}=0
$$



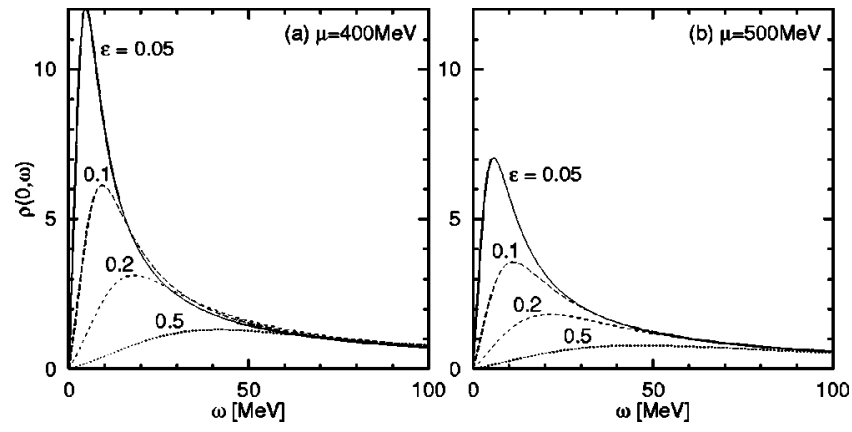

FIG. 2. The spectral function for the pair field at $T>T_{c}$ with $\epsilon$ $\equiv\left(T-T_{c}\right) / T_{c}=0.05,0.1,0.2$, and 0.5 at $\mu=400 \mathrm{MeV}$ (a) and $\mu$ $=500 \mathrm{MeV}(\mathrm{b})$.

owing to the self-consistency condition for the diquark condensate at $T=T_{c}$. This equality is the origin to lead to the various precritical phenomena for CSC, as we will see shortly. Here it should be stressed that although the following discussion seemingly relies heavily on the fact that phase transition is second order, the nature of the collective mode to be discussed below will not be altered even when the phase transition is weak first order.

The spectral function which is the measure of the excitation strength of the fluctuating pair field is given by

$$
\rho(\mathbf{k}, \omega)=-\frac{1}{\pi} \operatorname{Im} D^{R}(\mathbf{k}, \omega) .
$$

The temperature dependence of the spectral function at $\mu$ $=400 \mathrm{MeV}$ and zero momentum transfer $(\mathbf{k}=0)$ is given in Fig. 2(a). One can see that a prominent peak moving toward the origin grows as the temperature is lowered toward $T_{c}$, as indicated by the arrow in Fig. 1. From the experimental point of view, it is interesting that the peak survives at $T$ even well above $T_{c}$ with $\epsilon \equiv\left(T-T_{c}\right) / T_{c} \sim 0.2$. This means that the precritical region of CSC is one to two orders larger in the unit of $T_{c}$ than that in electric superconductors. Of course, this is related to the difference of the ratio $\omega_{D} / E_{F}$ and $\Lambda / E_{F}$, where $\omega_{D}$ is the Debye frequency and $E_{F}$ is the Fermi energy; the NJL model is similar to the attractive Hubbard model than to the BCS model [16]. The result for $\mu$ $=500 \mathrm{MeV}$ is shown in Fig. 2(b); one sees that although the growth of the peak becomes relatively moderate, the qualitative features do not change.

The existence of the peak with the narrow width suggests the existence of a well-defined collective mode as an elementary excitation in the Wigner phase. The existence and the dispersion relation $\omega=\omega(k)$ of a collective excitation in an isotropic system is examined by searching possible poles of the response function, i.e., solving the equation $1+G_{C} Q(k, \omega)=0$. Here $Q(k, \omega)$ is the analytic continuation of $\mathcal{Q}\left(k, \omega_{n}\right)$ to the upper half plane. The existence of a pole $\omega=\omega(k)$ for given $k$ means that the pair field $\Delta_{\text {ind }}(k, \omega(k))$ can be created even with an infinitesimal external field $\Delta_{e x}^{*}$ as seen from Eq. (6), which physically means that the system admits spontaneous excitation of a collective mode with the dispersion relation $\omega=\omega(k)$. Since the energy of the collective mode is found to be the lowest at $k=0$, let us obtain

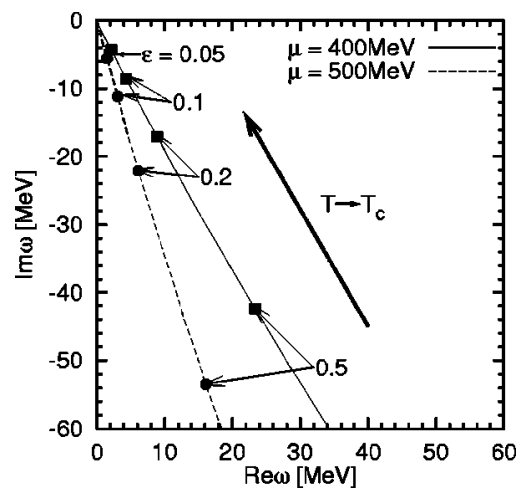

FIG. 3. The pole of the precursory soft mode for $\mu$ $=400 \mathrm{MeV}$ and $500 \mathrm{MeV}$. The numbers attached to dots denote $\boldsymbol{\epsilon}=\left(T-T_{c}\right) / T_{c}$.

$\omega(0) \equiv z$. One should notice that the gap equation (7) implies that $z=0$ is a solution at $T=T_{c}$, i.e., there exists a zero mode at the critical point. The singular behavior seen in the spectral function near the critical point in Fig. 2 is caused by the presence of the zero mode. Conversely, one may take the emergence of the zero mode as the critical condition for the color superconductivity. This condition is actually long known in the condensed matter physics and called the Thouless criterion [15].

Notice that the pole should be located in the lower half plane otherwise the system is unstable for the creation of the collective mode. Because of a cut along the real $z$ axis, the analytic continuation of $Q(0, z)$ to the lower half plane leads to the second Riemann sheet,

$$
\begin{aligned}
Q(0, z)= & \frac{2 N_{f}\left(N_{c}-1\right)}{\pi^{2}}\left[\mathrm{P} \int_{-\Lambda}^{\Lambda} k^{2} d k \frac{\tanh \frac{(k-\mu)}{2 T}}{z-2(k-\mu)}\right. \\
& \left.-\frac{1}{2} 2 \pi i\left(\mu+\frac{z}{2}\right)^{2} \tanh \frac{z}{4 T}\right]
\end{aligned}
$$

where $\mathrm{P}$ denotes the principal value and the term in the second line is the additional term for $z$ to be in the second sheet.

A numerical calculation shows that there indeed exists a pole in the lower half plane for $T>T_{c}$. Figure 3 shows how the pole moves as $T$ is lowered toward $T_{c}$ for $\mu=400$ and $500 \mathrm{MeV}$. One sees that the pole approaches the origin as $T$ is lowered toward $T_{c}$. Such a mode whose energy tends to vanish as the system approaches the critical point of a phase transition is called a soft mode [1]. Thus we have found the pole for the soft mode for CSC in hot quark matter. As far as we know, this is the first time when identification has been done of the softening pole in the complex energy plane for superconductivity, including condensed matter physics [15]. The significance of the existence of such a soft mode at $k$ $=0$ lies in the fact that it causes long-range correlations for the color Cooper pairs in the Wigner phase, which leads to a singular behavior of various observables such as the transport coefficients, as will be discussed later. We remark that 
such a soft mode in hot and dense quark matter was first discussed in [17] in relation to the chiral transition.

Another characteristic feature of the soft mode is that the absolute value of the imaginary part $\omega_{i}$ of the pole is larger than that of the real part $\omega_{r}$ for both $\mu$. This feature may have an important implication for an effective equation describing the dynamical phase transition for CSC: The dynamical behavior of the order parameter near $T_{c}$ is well described by a nonlinear diffusion equation which is known as the time-dependent Ginzburg-Landau (TDGL) equation [18]. We, however, remark that by setting $k=0$, the so-called Landau damping due to the particle-hole excitation is not incorporated in the present calculation. Figure 3 shows that the ratio $\omega_{i} / \omega_{r}$ becomes larger for the larger $\mu$. This is plausible because the larger the Fermi energy, the larger the density of states near the Fermi surface, which implies that the volume of the phase space for the decay of the pair field increases with $\mu$ at low $T$.

It is interesting that the so-called pseudogap in the density of states observed in high- $T_{c}$ superconductors (HTSC) as a characteristic precursory phenomenon [19] might be attributed to fluctuations of the Cooper pairs [20]. Thus the present results strongly suggest such a pseudogap phase exists also for CSC in a rather broad region of $T$ above $T_{c}$. To establish the pseudogap phase, one must calculate the density of states from the single particle Green's function $[16,20]$, which we hope to report on in a future publication. Experimentally, a measurement of the single-particle quark propagator is useful for obtaining the information on the density of states for the single quark states, as was done for HTSC by a kind of quasielastic scattering by photons, called the angleresolved photoemission spectroscopy (ARPES) [19].

The fluctuation of the pair field in metals above $T_{c}$ affects several transport coefficients like the electric conductivity (EC), the phonon absorption coefficient, and the specific heat [21]. An anomalous excess of the electric conductivity $T_{c}$ is known as paraconductivity (PC) [21]. The direct analogy thus leads to an expectation of the enhancement of color conductivity above $T_{c}$ in our case, which we call color paraconductivity (CPC): In fact, the PC for the metal superconductivity comes from the fact that the effective dynamics is given by the TDGL equation giving rise to a zero mode at momentum $k=0$ at $T=T_{c}$ [21], which is just the behavior extracted from the behavior of our soft mode as shown in Fig. 3.

More interesting is that the electric current can also have an anomalous enhancement in the Wigner phase due to the color pair-field fluctuations: This is because the propagator of photons (and also gluons) at $T>T_{c}$ is modified by the fluctuating pair field as in the usual superconductors [21]. This implies that photons and hence dileptons from the system can carry some information of the fluctuation of the color pair field.

In short, we have explored precursory phenomena for the color superconductivity (CSC) in quark matter at $T \neq 0$; the fluctuating pair field exists with a prominent strength even well above $T_{c}$. We have also discussed the possible observability of CSC through the fluctuations by heavy ion collisions with large baryon stopping using an analogy with the precritical phenomena known in the metal and the high- $T_{c}$ superconductors.

We thank M. Alford for his interest in our work and for suggesting the possible relevance of our work to [22]. O. Tchernyshyov is gratefully acknowledged for his encouragement and useful comments on the relevance of the pseudogap phenomena in condensed matter physics [23] to our case. We are indebted to M. Asakawa for indicating a misleading statement present in the original manuscript. T. Kunihiro was partially supported by the Grants-in-Aid of the Japanese Ministry of Education, Science and Culture (Nos. 12640263 and 12640296).
[1] T. Hatsuda and T. Kunihiro, Phys. Rep. 247, 221 (1994).

[2] G.E. Brown and M. Rho, Phys. Rep. 269, 333 (1996).

[3] For example, see F. Karsch, hep-lat/0106019; Z. Fodor and S.D. Katz, J. High Energy Phys. 03, 014 (2002).

[4] See, for example, U. Vogl and W. Weise, Prog. Part. Nucl. Phys. 27, 195 (1991); R. Alkofer and L. von Smekal, Phys. Rep. 353, 281 (2001), and the references therein.

[5] B. Barrios, Nucl. Phys. B129, 390 (1977); D. Bailin and A. Love, Phys. Rep. 107, 325 (1984); M. Iwasaki and T. Iwado, Prog. Theor. Phys. 94, 1073 (1995).

[6] R. Rapp, T. Schäfer, E. Shuryak, and M. Velkovsky, Phys. Rev. Lett. 81, 53 (1998); M. Alford, K. Rajagopal, and F. Wilczek, Phys. Lett. B 422, 247 (1998).

[7] D.T. Son, Phys. Rev. D 59, 094019 (1999); R.D. Pisarski and D.H. Rischke, Phys. Rev. Lett. 83, 37 (1999); T. Schaffer and F. Wilczek, Phys. Rev. D 60, 114033 (1999).

[8] K. Rajagopal and F. Wilczek, in Handbook of $Q C D$, edited by M. Shifman (World Scientific Singapore, 2001); M. Alford, Annu. Rev. Nucl. Part. Sci. 51, 131 (2001).
[9] See Proceedings of the GSI Workshop on its Future Facility: http://www.gsi. de/GSI-Future/

[10] J. Berges and K. Rajagopal, Nucl. Phys. B538, 215 (1999).

[11] T.M. Schwarz, S.P. Klevansky, and G. Papp, Phys. Rev. C 60, 055205 (1999).

[12] Y. Nambu and G. Jona-Lasinio, Phys. Rev. 122, 345 (1961); 124, 246 (1961).

[13] S.P. Klevansky, Rev. Mod. Phys. 64, 649 (1992).

[14] D. Ebert, H. Reinhardt, and M.K. Volkov, Prog. Part. Nucl. Phys. 33, 1 (1994).

[15] D.J. Thouless, Ann. Phys. (N.Y.) 10, 553 (1960).

[16] See, for example, V.M. Loktev, R.M. Quick, and S.G. Sharapov, Phys. Rep. 349, 1 (2001).

[17] T. Hatsuda and T. Kunihiro, Phys. Rev. Lett. 55, 158 (1985); Phys. Lett. 145B, 7 (1984).

[18] M. Cyrot, Rep. Prog. Phys. 36, 103 (1973).

[19] As a review, T. Timusk and B. Statt, Rep. Prog. Phys. 62, 61 (1999). 
[20] I. Kosztin et al., Phys. Rev. B 61, 11662 (2000) and references therein; see for the pseudogap phenomena in other fields, E. Babaev, Int. J. Mod. Phys. A 16, 1175 (2001); A. Schnell, G. Röpke, and P. Schuck, Phys. Rev. Lett. 83, 1926 (1999).

[21] L.G. Aslamazov and A.I. Larkin, Fiz. Tverd. Tela (Leningrad) 10, 1104 (1968) [Sov. Phys. Solid State 10, 875 (1968)]; K.
Maki, Prog. Theor. Phys. 40, 193 (1968); R.S. Thompson, Phys. Rev. B 1, 327 (1970).

[22] B. Bordnikov and K. Rajagopal, Phys. Rev. D 61, 105017 (2000).

[23] O. Tchernyshyov, Phys. Rev. B 56, 3372 (1997). 